\title{
РЕЗУЛЬТАТЫ РАЗРАБОТКИ «ЭЛЕКТРОННОЙ» ПАЛЕТКИ ДЛЯ ВЫЧИСЛЕНИЯ УКЛОНЕНИЯ ОТВЕСНОЙ ЛИНИИ
}

\author{
Денис Николаевич Голдобин \\ Сибирский государственный университет геосистем и технологий, 630108, Россия, г. Ново- \\ сибирск, ул. Плахотного, 10, кандидат технических наук, старший преподаватель кафедры \\ космической и физической геодезии, тел. (913)455-92-66, e-mail: phis.geo.sgga@gmail.com
}

\section{Вадим Федорович Канушин}

Сибирский государственный университет геосистем и технологий, 630108, Россия, г. Новосибирск, ул. Плахотного, 10, кандидат технических наук, доцент кафедры космической и физической геодезии, тел. (913)983-77-26, e-mail: kanushinvf43.work.ssg@gmail.com

\section{Ирина Геннадьевна Ганагина}

Сибирский государственный университет геосистем и технологий, 630108, Россия, г. Новосибирск, ул. Плахотного, 10, кандидат технических наук, зав. кафедрой космической и физической геодезии, тел. (913)956-46-42, e-mail: gam0209@yandex.ru

Дано описание разработанной «электронной» палетки, применяемой для автоматического снятия данных с цифровой модели аномалий силы тяжести с помощью функции Digitize для вычисления гравиметрических уклонений отвесной линии (УОЛ). В статье проведено сравнение результатов вычисления гравиметрических УОЛ с помощью «электронной» палетки и с помощью обычных палеток путем «ручной» обработки данных с результатами определения гравиметрических УОЛ на 15 пунктах Лапласа, расположенных на территории Горного Алтая. Показано, что автоматический режим получения УОЛ разработанной «электронной» палеткой позволяет достичь высокой точности обработки результатов при высокой скорости записи данных. Применение круговых «электронных» палеток рекомендуется использовать в методических целях для определения гравиметрических уклонений отвесной линии.

Ключевые слова: уклонения отвесной линии, «электронная» палетка, модель геопотенциала EIGEN-6C4, гравиметрические и астрономо-геодезические уклонения

\section{RESULTS OF THE DEVELOPMENT OF AN ELECTRONIC PACKAGE FOR CALCULATING THE PLANE LINE DEPLOYMENT}

\section{Denis N. Goldobin}

Siberian State University of Geosystems and Technologies, 10, Plakhotnogo St., Novosibirsk, 630108, Russia, Ph. D., Senior Lecturer, Department of Space and Physical Geodesy, phone: (913)455-92-66, e-mail: phis.geo.sgga@gmail.com

\section{Vadim F. Kanushin}

Siberian State University of Geosystems and Technologies, 10, Plakhotnogo St., Novosibirsk, 630108, Russia, Ph. D., Associate Professor, Department of Space and Physical Geodesy, phone: (913)983-77-26, e-mail: kanushinvf43.work.ssg@gmail.com

\section{Irina G. Ganagina}

Siberian State University of Geosystems and Technologies, 10, Plakhotnogo St., Novosibirsk, 630108, Russia, Ph. D., Head of the Department of Space and Physical Geodesy, phone: (913)956-46-42, e-mail: gam0209@yandex.ru 
A description of the developed "electronic" palette is given, which is used for automatic data collection from a digital model of gravity anomalies using the Digitize function to calculate the gravimetric deviations of the plumb line (DPL). The article compares the results of calculating the gravimetric using an "electronic" pallet and using conventional pallets by "manual" data collection with the results of determining the gravimetric DPL at 15 Laplace points located in the Altai Mountains. It is shown that the automatic mode with the help of the developed "electronic" palette, showed itself better from all sides, this is also the higher the accuracy and speed of data recording. The use of circular "electronic" pallets is recommended for educational purposes, to consolidate knowledge of determining the gravimetric deviations of the plumb line.

Keywords: plumb line deviations, "electronic" palette, EIGEN-6C4 geopotential model, gravimetric and astronomical-geodetic deviations

\section{Введение}

УОЛ является одной из важных характеристик геометрической структуры гравитационного поля Земли. Данные об УОЛ необходимы для решения редукционных задач геодезии и требуются, в частности, для высокоточной автономной навигации, где неточное знание УОЛ искажает показания инерциальных навигационных систем (ИНС). УОЛ содержит в себе информацию о напряженности и распределении аномальных масс в земной коре, которая необходима при решении задач геофизики и геодинамики. Проблема определения уклонений отвесных линий (УОЛ) актуальна для всех регионов Земли [1-5].

Уклонение отвесной линии u, определяется по формуле

$$
u=\xi \cos A+\eta \sin A,
$$

где $\xi$ и $\eta$ - составляющие УОЛ в плоскости меридиана и плоскости первого вертикала соответственно; $A$-азимут плоскости полного уклонения отвеса $u$.

Традиционные методы определения уклонений отвесных линий сложны и трудоемки. Астрономо-геодезический метод требует выполнения высокоточных полевых астрономических и геодезических наблюдений. В этом методе составляющие уклонения отвеса $\xi$ и $\eta$ в формуле (1) можно определить в результате сравнения астрономических координат $\varphi$ и $\lambda$ с геодезическими B и L на пунктах геодезических сетей.

Гравиметрический метод вычисления уклонений отвесных линий предполагает наличие детальной гравиметрической съемки на всей земной поверхности и трудоемкий процесс определения УОЛ как результат считывания информации по гравиметрическим картам.

В работе представлена разработанная авторами «электронная» палетка, которая автоматизирует процесс получения информации с цифровой модели аномалий силы тяжести с помощью функции Digitize для вычисления гравиметрических уклонений отвесной линии. Выполнено сравнение результатов вычисле- 
ния гравиметрических УОЛ с помощью «электронной» палетки и с помощью обычных палеток путем «ручной» обработки данных с результатами определения гравиметрических УОЛ на 15 пунктах Лапласа, расположенных на территории Горного Алтая.

\section{Методы и материалы}

В настоящее время измеряемые параметры гравитационного поля Земли (точечные аномалии силы тяжести) известны с высоким разрешением и высокой точностью до (1-5) $10^{-8} \mathrm{Mc}^{-2}$ лишь на ограниченных участках с плотной гравиметрической съемкой. Причем эти участки распределены на земном шаре неравномерно. Почти на $30 \%$ земной поверхности измерения силы тяжести отсутствуют [6]. Поэтому прибегают к локальному описанию гравитационного поля, которое необходимо при создании опорных геодезических сетей для решения задач высшей и прикладной геодезии, геофизики и геодинамики в масштабе государства, континента и прилегающих к ним акваторий морей и океанов или небольшого участка, в виде стройплощадки [7]. Использование гравиметрического метода при детальных местных и региональных исследованиях фигуры и внешнего гравитационного поля Земли связано с успешным решением краевых задач физической геодезии как в Стоксовом приближении, так и в соответствии с теорией М. С. Молоденского [8]. В работе [9] нахождение возмущающего потенциала Т на физической поверхности Земли сведено к решению третьей краевой задачи с краевым условием на границе $\Sigma$

$$
\left(\frac{\partial T}{\partial \rho}\right)_{\Sigma}+\left(\frac{2 T}{\rho}\right)_{\Sigma}=-g_{S}+\gamma_{\Sigma},
$$

где индексы $\Sigma$ и $\mathrm{S}$ означают величины относящиеся к поверхности Земли в первом приближении $\Sigma$ и к ее физической поверхности S.

В качестве исходной уровенной поверхности нормального потенциала берется сфера радиуса R. В этом случае радиус-вектор точки на поверхности $\Sigma$ будет равен

$$
\rho_{\Sigma}=R+H^{\gamma}
$$

где $H^{\gamma}$ нормальная высота тачки на поверхности $\Sigma$.

$\mathrm{B}$ результате решение уравнения Лапласа $\Delta \mathrm{T}=0$ для сферы $\omega$ радиуса $\mathrm{R} \mathrm{c}$ краевым условием (2) принимает следующий вид:

$$
T_{p}=\frac{R}{4 \pi} \iint_{\omega} \Delta g_{M} S(\psi) d \omega,
$$

где $T_{p}$ - возмущающий потенциал в точке $P(\varphi, \lambda)$; 
$\Delta g_{M}$ - аномалия силы тяжести в свободном воздухе в точке $\mathrm{M}\left(\varphi^{\prime}, \lambda\right) ; \psi$ сферического расстояние между точками $P(\varphi, \lambda)$ и $\mathrm{M}\left(\varphi^{\prime}, \lambda\right)$ определяется по следующей формуле:

$$
\cos \psi=\sin \varphi \sin \varphi^{\prime}+\cos \varphi \cos \varphi^{\prime} \cos \left(\lambda-\lambda^{\prime}\right) .
$$

Функция $S(\psi)$ функция Стокса, она равна

$$
S(\psi)=\sum_{n=2}^{\infty} \frac{2 n+1}{n-1} P_{n}(\cos \psi)
$$

где $P_{n}(\cos \psi)$ - полином Лежандра степени п.

Используя формулу Брунса [10], получим высоту геоида по формуле:

$$
\zeta_{p}=\frac{T_{p}}{\gamma}
$$

или с учетом выражения (4) получим

$$
\zeta_{p}=\frac{R}{4 \pi \gamma} \iint_{\omega} \Delta g_{M} S(\psi) d \omega
$$

Поскольку значения $\xi, \eta$ малы, то для вычисления составляющих УОЛ в пункте с координатами $\varphi, \lambda$ можно воспользоваться следующими дифференциальными соотношениями

$$
\begin{gathered}
\xi^{\prime \prime}=-\frac{\rho^{\prime \prime}}{M} \cdot \frac{\partial \zeta}{\partial \varphi}, \\
\eta^{\prime \prime}=-\frac{\rho^{\prime \prime}}{N \cdot \cos \varphi} \cdot \frac{\partial \zeta}{\partial \lambda} .
\end{gathered}
$$

В формулах (8)-(9) $M$ - радиус кривизны в меридиане, $N$ - радиус кривизны в первом вертикале.

Радиус кривизны в меридиане определяется соотношением:

$$
M=\frac{a \cdot\left(1-e^{2}\right)}{\left(1-e^{2} \cdot \sin ^{2} \varphi\right)^{3 / 2}},
$$


а радиус кривизны в первом вертикале

$$
N=\frac{a}{\left(1-e^{2} \cdot \sin ^{2} \varphi\right)^{1 / 2}} .
$$

В формулах (10)-(11) $a, e-$ большая полуось и эксцентриситет отсчетного эллипсоида, соответственно.

После дифференцирования выражения (7) в формуле (8) по широте, а в формуле (9) по долготе, получены формулы Венинг-Мейнесса

$$
\left.\begin{array}{l}
\xi_{p}^{\prime \prime}=\frac{1}{2 \pi} \int_{0}^{\pi} \int_{0}^{2 \pi} \Delta g Q(\psi) \cos A d A d \psi \\
\eta_{p}^{\prime \prime}=\frac{1}{2 \pi} \int_{0}^{\pi} \int_{0}^{2 \pi} \Delta g Q(\psi) \sin A d A d \psi
\end{array}\right\}
$$

где $\Delta g$ - аномалия силы тяжести;

А - азимут плоскости полного УОЛ;

$$
Q(\psi)=-\frac{\rho^{\prime \prime}}{2 \gamma} \cos ^{2} \frac{\psi}{2}\left[\begin{array}{l}
\operatorname{cosec} \frac{\psi}{2}+12 \sin \frac{\psi}{2}-32 \sin ^{2} \frac{\psi}{2}+\frac{3}{1+\sin \frac{\psi}{2}}- \\
-12 \sin ^{2} \frac{\psi}{2} \ln \left(\sin \frac{\psi}{2}+\sin ^{2} \frac{\psi}{2}\right)
\end{array}\right] .
$$

Формула (13) - функция Венинг-Мейнесса. В табл. 1 приведены значения функции Венинг-Мейнесса, вычисленные по формуле (13).

Таблищза 1

Значения функции Венинг-Мейнесса

\begin{tabular}{|c|c|c|c|c|c|}
\hline$\psi$ & $\mathrm{Q}$ & $\psi$ & $\mathrm{Q}$ & $\psi$ & $\mathrm{Q}$ \\
\hline 0 & & 60 & $+0,22$ & 130 & $-0,34$ \\
\hline 1 & $+12,35$ & 70 & $+0,03$ & 140 & $-0,24$ \\
\hline 10 & $+1,59$ & 80 & $-0,15$ & 150 & $-0,16$ \\
\hline 20 & $+1,02$ & 90 & $-0,29$ & 160 & $-0,08$ \\
\hline 30 & $+0,79$ & 100 & $-0,38$ & 170 & $-0,02$ \\
\hline 40 & $+0,61$ & 110 & $-0,41$ & 180 & 0,00 \\
\hline 50 & $+0,43$ & 120 & $-0,40$ & & \\
\hline
\end{tabular}


В формулах (12) интегрирование должно выполняться по всей поверхности $\omega$ сферической Земли, то есть предполагается, что аномалии силы тяжести в свободном воздухе $\Delta g$ известны на ней в виде непрерывной функции. Однако из таблицы 1 видно, что с увеличением сферического расстояния $\psi$ от исследуемого пункта $P$ до текущей точки $M$ на поверхности сферы $\omega$ функция $Q(\psi)$ быстро убывает. Поэтому аномалии силы тяжести в отдаленных пунктах могут быть известны с меньшей точностью, чем областях близких к исследуемой точке. Функция $Q(\psi)$ быстро убывает в пределах первых $10^{\circ}$, а от $\psi=20^{\circ}$ до $\psi=$ $180^{\circ}$ близка к нулю. Такое поведение весовой функции позволяет разделить область интегрироания на две части. В настоящее время первую часть ограничивают радиусом 50 км, в которой вычисление высот геоида и уклонений выполняется вручную с помощью палетки, рассчитанной в ЦНИИГАиК В. Ф. Еремееым [11]. Для учета влияния второй части области интегрирования радиусом более 50 км настоящее время используются современные глобальные модели гравитационного поля. При определении уклонений отвеса основное значение имеют плоские зоны, обычно радиусом порядка 100 км.

\section{Результаты}

Используя аномалии силы тяжести в свободном воздухе, полученные с погрешностью 3 мГал с помощью методов спутниковой гравиметрии $[12,13]$ на 15 пунктах Лапласа на участке горного Алтая вычислены составляющие уклонения отвеса. Картосхема которых приведена на рис.1

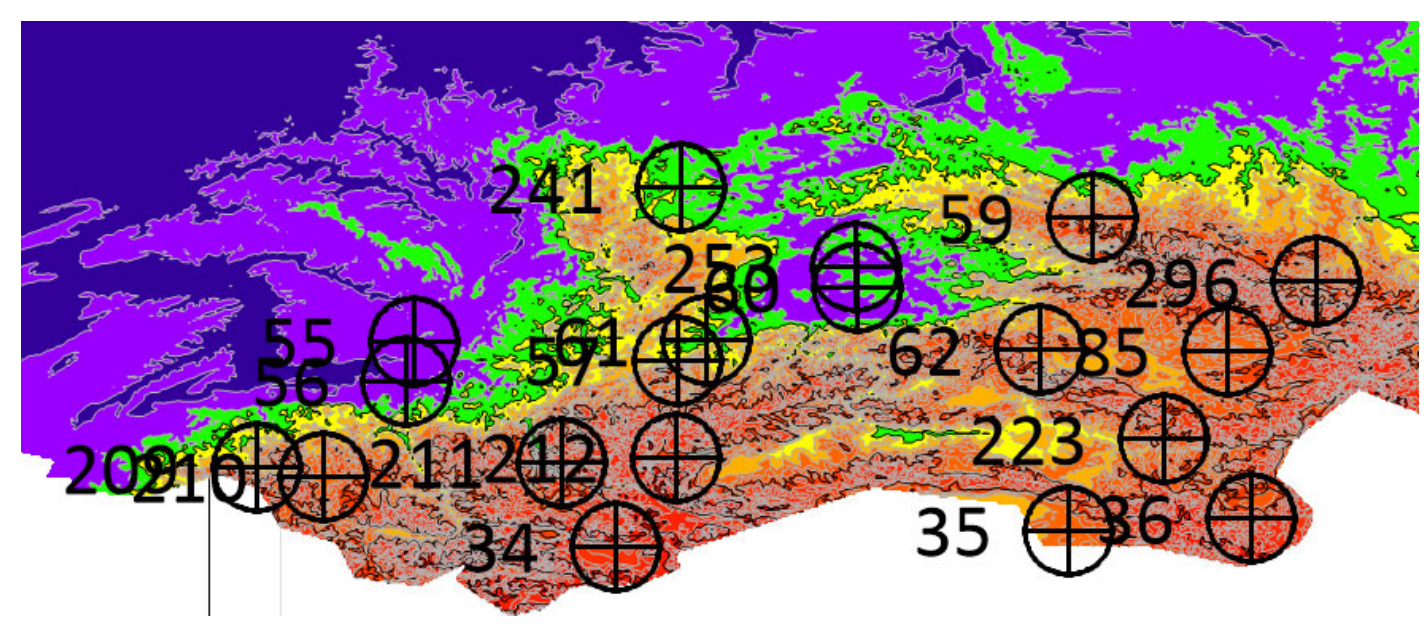

Рис. 1. Пункты Лапласа на участке горного Алтая (расположение палетки обозначено кружками радиусом 100км; цифры - номера пунктов)

Для вычисления УОЛ палетка, разработанная в ЦНИИГАиК В. Ф. Еремеевым [11], была преобразована для работы на персональном компьютере в виде «Электронной палетки», которая приведена на рис. 2. 


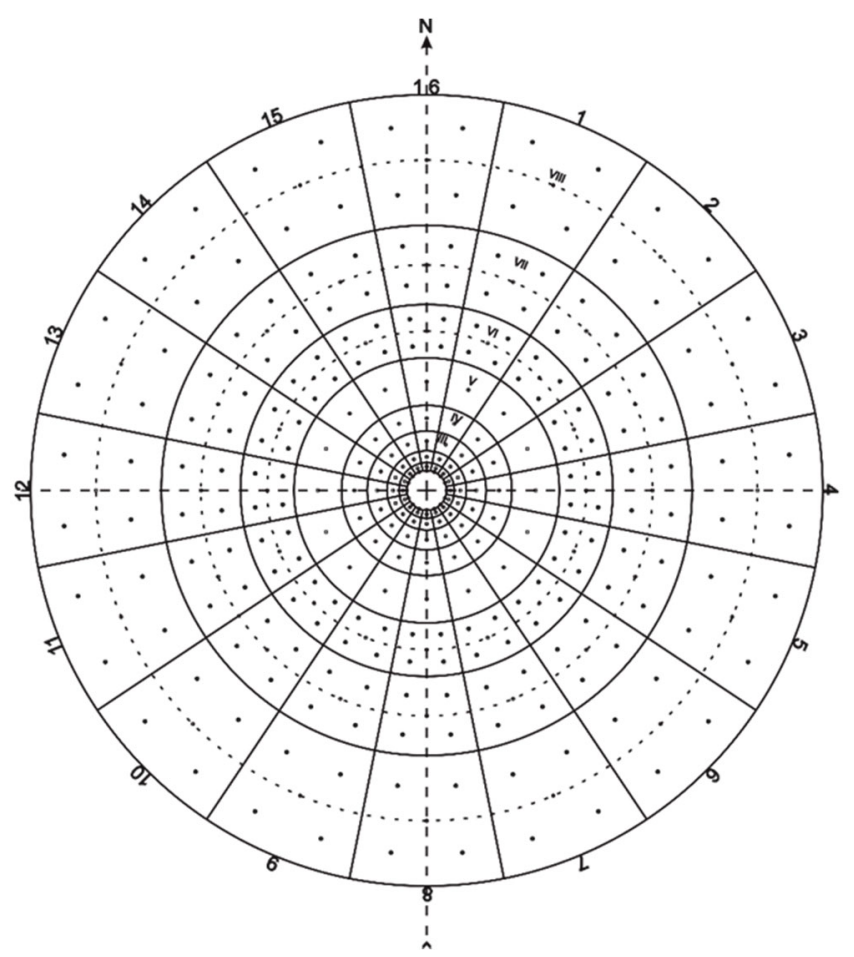

Рис. 2. «Электронная палетка»

Вычисления уклонения отвесной линии выполняется по формулам ВенингМейнеса (12), используя записанные в цифровом виде значения в точках по палетке (рис. 3).

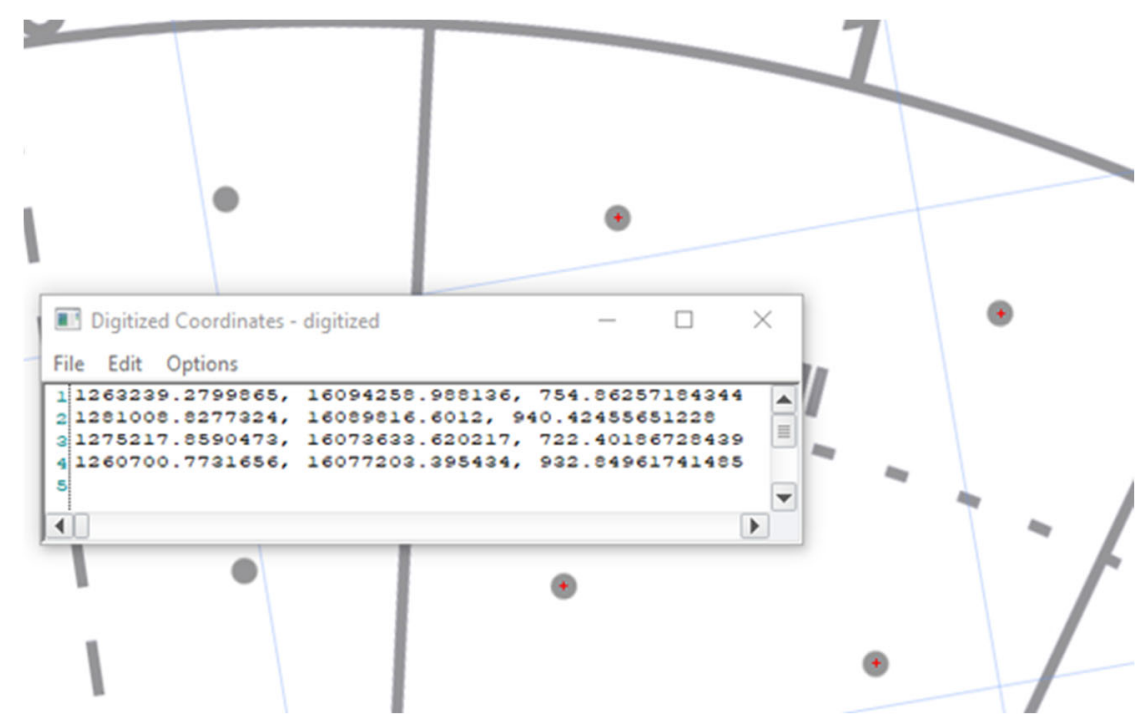

Рис. 3. Выбор точек и фиксация их координат

Результаты вычисления гравиметрических УОЛ с помощью «Электронной палетки» приведены в табл. 2. 
Таблицуа 2

Результаты вычисления гравиметрических составляющих УОЛ на территории горного Алтая

\begin{tabular}{|c|c|c|}
\hline № точек & $\xi^{\prime \prime}$ & $\eta^{\prime \prime}$ \\
\hline 34 & 2,54 & 1,12 \\
\hline 35 & 1,33 & 1,05 \\
\hline 36 & $-0,46$ & $-0,79$ \\
\hline 57 & 3,37 & $-0,06$ \\
\hline 61 & $-1,25$ & 0,39 \\
\hline 62 & $-2,95$ & $-0,49$ \\
\hline 85 & $-2,78$ & $-0,8$ \\
\hline 169 & 3,06 & $-2,42$ \\
\hline 170 & $-2,98$ & 1,62 \\
\hline 209 & $-1,99$ & 1,85 \\
\hline 210 & 0,16 & 1,5 \\
\hline 211 & $-4,13$ & $-0,8$ \\
\hline 212 & 0,85 & $-0,75$ \\
\hline 223 & 3,45 & $-0,7$ \\
\hline 296 & $-3,79$ & $-3,98$ \\
\hline
\end{tabular}

В таблице 3 приведены результаты сравнения астрономо-геодезических составляющих УОЛ полученных по формулам (7) и (8) на пунктах Лапласа в горном Алтае с гравиметрическими составляющих УОЛ, вычисленными с помощью «Электронной» палетки при учете аномалий силы тяжести в радиусе 5км и 100км.

Таблицьа 3

Статистические характеристики распределения результатов сравнения гравиметрических уклонений отвеса вычисленными с помощью «Электронной» палетки с астрономо-геодезическими в горном районе Алтая

\begin{tabular}{|c|c|c|c|c|c|c|c|c|}
\hline Обозначения & $\xi "$ & $\eta "$ & $\xi "$ & $\eta^{\prime \prime}$ & $\xi "$ & $\eta "$ & $\xi "$ & $\eta^{\prime \prime}$ \\
\hline Учитываемый радиус & \multicolumn{2}{|c|}{5 км } & \multicolumn{2}{|c|}{$5 \kappa \mathrm{M}$} & \multicolumn{2}{|c|}{100 км } & \multicolumn{2}{|c|}{100 км } \\
\hline$\rho$ & \multicolumn{2}{|c|}{$2,67 \Gamma / \mathrm{cm}^{3}$} & \multicolumn{2}{|c|}{$2,5 \Gamma / \mathrm{cm}^{3}$} & \multicolumn{2}{|c|}{$2,67 \Gamma / \mathrm{cm}^{3}$} & \multicolumn{2}{|c|}{$2,5 \Gamma / \mathrm{cm}^{3}$} \\
\hline Количество точек & 15 & 15 & 15 & 15 & 15 & 15 & 15 & 15 \\
\hline Минимальная разность & $-9,15$ & $-2,77$ & $-9,06$ & $-2,78$ & $-1,61$ & $-5,03$ & $-1,76$ & $-4,24$ \\
\hline Максимальная разность & 11,32 & 5,62 & 11,6 & 5,73 & 7,53 & 4,28 & 7,63 & 3,87 \\
\hline Диапазон & 20,47 & 8,39 & 20,66 & 8,51 & 9,14 & 9,31 & 9,39 & 8,11 \\
\hline Средняя & $-0,28$ & 0,95 & $-0,26$ & 0,96 & 2,44 & $-0,37$ & 2,29 & $-0,28$ \\
\hline Дисперсия & 45,59 & 6,15 & 46,36 & 6,14 & 7,35 & 5,27 & 8,15 & 4,12 \\
\hline Стандартное отклонение & 6,75 & 2,48 & 6,81 & 2,48 & 2,71 & 2,30 & 2,85 & 2,03 \\
\hline
\end{tabular}

В табл. 4 приведены статистические характеристики результатов сравнения гравиметрических уклонений отвеса с астрономо-геодезическими в горном районе Алтая. 
Статистические характеристики распределения результатов сравнения гравиметрических уклонений отвеса с астрономо-геодезическими в горном районе Алтая

\begin{tabular}{|c|c|c|c|c|c|c|c|c|}
\hline Обозначения & $\xi "$ & $\eta^{\prime \prime}$ & $\xi "$ & $\eta^{\prime \prime}$ & $\xi^{\prime \prime}$ & $\eta^{\prime \prime}$ & $\xi^{\prime \prime}$ & $\eta^{\prime \prime}$ \\
\hline Учитываемый радиус & \multicolumn{2}{|c|}{5 Км } & \multicolumn{2}{|c|}{$5 \kappa \mathrm{M}$} & \multicolumn{2}{|c|}{100 км } & \multicolumn{2}{|c|}{100 км } \\
\hline$\rho$ & \multicolumn{2}{|c|}{$2,67 \Gamma / \mathrm{cm}^{3}$} & \multicolumn{2}{|c|}{$2,5 \Gamma / \mathrm{cm}^{3}$} & \multicolumn{2}{|c|}{$2,67 \Gamma / \mathrm{cm}^{3}$} & \multicolumn{2}{|c|}{$2,5 \Gamma / \mathrm{cm}^{3}$} \\
\hline Количество точек & 15 & 15 & 15 & 15 & 15 & 15 & 15 & 15 \\
\hline Минимальная разность & $-9,15$ & $-2,77$ & $-9,06$ & $-2,78$ & $-1,61$ & $-5,03$ & $-1,76$ & $-4,24$ \\
\hline Максимальнс & 11,32 & 5,62 & 11,60 & 5,73 & 7,53 & 4,28 & 7,63 & 3,87 \\
\hline Диапазон & 20,47 & 8,39 & 20,66 & 8,50 & 9,14 & 9,30 & 9,38 & 8,11 \\
\hline Среді & $-0,28$ & 0,95 & $-0,26$ & 0,96 & 2,44 & $-0,37$ & 2,29 & $-0,28$ \\
\hline Дисперсия & 48,84 & 6,58 & 49,68 & 6,57 & 7,87 & 5,65 & 8,72 & 4,41 \\
\hline Стандартное отклонение & 6,99 & 2,56 & 7,05 & 2,56 & 2,81 & 2,38 & 2,95 & 2,10 \\
\hline
\end{tabular}

Из таблицы 4 видно, что минимальное стандартное отклонение по $\xi$ составляет 2,81" при плотностях 2,67" при 100 км, а $\eta 2,10 "$ при плотности 2,5" при 100 км. Средние значения для них составляют 2,44" и -0,28".

\section{Обсуждение}

Следует отметить некоторые недостатки применения круговых палеток для определения УОЛ гравиметрическим методом. Во-первых, радиусы кольцевых зон необходимые для учета, как отмечено работе [Моловичко], определены недостаточно точно. Во-вторых, палетки поострены без учета картографических искажений, которые выражаются величинами, значительно превышающими точность графических построений особенно при увеличении радиуса палетки. В-третьих, аномалии силы тяжести $\Delta \mathrm{g}$, строго говоря, относятся к физической поверхности $\mathrm{S}$, а интегрирование выполняется по упрощенным формулам на горизонтальной плоскости и, следовательно, аномалии должны быть отнесены к этой плоскости.

В результате использования двух методов сбора гравиметрических данных: «ручной» с цифровой карты и запись данных в автоматическом режиме с помощью «электронной» палетки показали, что стандартные отклонения на всех радиусах учета аномалий силы тяжести меньше при использовании «электронной» палетки.

Бумажные носители со временем приходят в негодность и требуют затрат на восстановление и поддержание их в рабочем состоянии. Данного недостатка лишена методика работы с «электронной» палеткой, которая не изнашивается, а создание более точных цифровых моделей и внедрение их в рабочий процесс гораздо проще.

\section{БИБЛИОГРАФИЧЕСКИЙ СПИСОК}

1. Конешов, В.Н. Оценка навигационной информативности аномального гравитационного поля Земли [Текст] / В.Н. Конешов, В.Б. Непоклонов, Л.И. Августов // Гироскопия и навигация. -2016 . - №2 (93). - С. $95-106$.

2. Рожков, Ю.А. О возможности применения результатов аэрогравиметрических измерений для вычисления уклонений отвесной линии в труднодоступных районах [Текст] / Ю.А. Рожков, Н.В. Дробышев, В.Н. Конешов, В.В. Клевцов // Физика Земли. - 2005. - № 2. - С. 84 - 87. 
3. Алексеев С.П. Современные направления реализации технологий навигационногидрографического и гидрометеорологического обеспечения морской деятельности/Доклад на пятой Российской научно технической конференции "Современное состояние и проблемы навигации и океанографии ИНФОРМОСТ радиоэлектроника и телекоммуникации № 2 (32) 2004,c. 30-32.

4. Конешов, В.Н. Методика расчета уклонения отвесной линии на основе Sаппроксимаций [Текст] / В.Н. Конешов, И.В. Осика, И.Э. Степанова // Физика Земли. - 2007. - № 6. - С. 19 - 25.

5. Боярский, Э.А. К вычислению уклонений отвесной линии и превышений геоида по гравитационным аномалиям [Текст] / Э.А. Боярский, Л.В. Афанасьева, В.Н. Конешов, Ю.А. Рожков // Физика Земли. - 2010. - № 6. - С. 80 - 85.

6. Торге Вольфганг. Гравиметрия/Пер. с англ. -М., Мир, 1999. -429с.

7. Бровар, В.В. Граитационное поле в задачах инженерной геодезии[Текст] /В.В. Бровар. М., Недра, 1983, 112с.

8. Молоденский, М. С. Основные вопросы геодезической гравиметрии[Текст] / Тр. ЦНИИГАиК, вып. 42. 1945. 106 с.

9. Молоденский, М. С. Методы изучения внешнего гравитационного поля и фигуры Земли. [Текст] /М.С. Молоденский, В.Ф. Еремеев, М.И. Юркина// Тр. ЦНИИГАиК, 1960, вып. 131.251с.

10. Огородова Л. В. Нормальное поле и определение аномального потенциала: Учебное пособие. -М.: Изд-во МИИГАиК, 2010. -105 с.

11. Еремеев, В. Ф. Расчет палетки для вычисления высот квазигеоида и уклонений отеса по формулам Стокса и Венинг-Мейнеса. [Текст] / В.Ф. Еремеев // Тр. ЦНИИГАиК, 1957, вып. 121. М., Геодезиздат, с.43-75.

12. Кащеев Р.А. Современные методы спутниковой гравиметрии Конспект лекций/ Р.А.Кащеев. - Казань: Казан.ун-т, 2015. - 45с. ],

13. Исследование современных глобальных моделей гравитационного поля Земли [Текст] : монография / В. Ф. Канушин, А. П. Карпик, И. Г. Ганагина, Д. Н. Голдобин, А. М. Косарева, Н. С. Косарев - Новосибирск: СГУГиТ, 2015. - 270 с.

(C) Д. Н. Голдобин, В. Ф. Канушин, И. Г. Ганагина, 2021 